Appl. Set-Valued Anal. Optim. 4 (2022), No. 1, pp. 95-107

Available online at http://asvao.biemdas.com

https://doi.org/10.23952/asvao.4.2022.1.07

\title{
OPTIMAL SOLUTIONS TO VARIATIONAL INEQUALITIES VIA BREGMAN DISTANCES IN BANACH LATTICES
}

\author{
ESKANDAR NARAGHIRAD
}

Department of Mathematics, Yasouj University, Yasouj 75918, Iran

\begin{abstract}
We study the existence of maximum and minimum solutions to generalized variational inequalities on Banach lattices. The main tools are the variational characterization of the Bregman generalized projection and the order-theoretic fixed point theory.
\end{abstract}

Keywords. Banach lattices; Bregman generalized projection operators; Fixed points; Variational inequalities.

\section{INTRODUCTION}

Throughout this paper, we denote the set of real numbers and the set of natural numbers by $\mathbb{R}$ and $\mathbb{N}$, respectively. Let $E$ be a Banach space and $E^{*}$ its dual space. If $x \in E$, then we denote the value of $x^{*} \in E^{*}$ at $x$ by $\left\langle x, x^{*}\right\rangle$. For any sequence $\left\{x_{n}\right\}_{n \in \mathbb{N}}$ in $E$, we denote the strong convergence of $\left\{x_{n}\right\}_{n \in \mathbb{N}}$ to $x \in E$ by $x_{n} \rightarrow x$ and the weak convergence by $x_{n} \rightarrow x$ as $n \rightarrow \infty$. To see some geometric properties of Banach spaces, we refer the reader to [1]. Let $C$ be a nonempty subset of a Banach space $E$. Let $f: C \rightarrow E$ be a map. We denote by Fix $(f)=\{x \in C: f x=x\}$ the set of fixed points of $f$. Let $X$ be a topological space. In the sequel, we denote the set of all nonempty subsets of $X$ by $P(X)$.

We recall the definition of the Bregman distances. Let $E$ be a Banach space and $g: E \rightarrow \mathbb{R}$ be a strictly convex and Gâteaux differentiable function on a Banach space $E$. The Bregman distance [2] (see also [3, 4]) corresponding to $g$ is the function $D_{g}: E \times E \rightarrow \mathbb{R}$ defined by

$$
D_{g}(x, y)=g(x)-g(y)-\langle x-y, \nabla g(y)\rangle, \quad \forall x, y \in E .
$$

It is clear that $D_{g}(x, y) \geq 0$ for all $x, y$ in $E$. However, $D_{g}$ is not symmetric, and $D_{g}$ does not satisfy the triangular inequality. When $E$ is a smooth Banach space, setting $g(x)=\|x\|^{2}$ for all $x$ in $E$, we have that $\nabla g(x)=2 J x$ for all $x$ in $E$. Here $J$ is the normalized duality mapping from $E$ into $E^{*}$. Hence, $D_{g}(\cdot, \cdot)$ reduces to the usual map $\phi(\cdot, \cdot)$ as

$$
D_{g}(x, y)=\phi(x, y):=\|x\|^{2}-2\langle x, J y\rangle+\|y\|^{2}, \quad \forall x, y \in E .
$$

Let $E$ be a Banach space and $g: E \rightarrow \mathbb{R}$ a strictly convex and Gâteaux differentiable function. Working on the Bregman distance $D_{g}$ (see (1.1)), the following Bregman three-point identity

E-mail addresses: eskandarrad@gmail.com; esnaraghirad@yu.ac.ir.

Received September 9, 2021; Accepted November 5, 2021.

(C)2022 Applied Set-Valued Analysis and Optimization 
holds for every Banach space $E$ [4]:

$$
D_{g}(x, z)=D_{g}(x, y)+D_{g}(y, z)+\langle x-y, \nabla g(y)-\nabla g(z)\rangle, \quad \forall x, y, z \in E .
$$

In particular,

$$
D_{g}(x, y)=-D_{g}(y, x)+\langle y-x, \nabla g(y)-\nabla g(x)\rangle, \quad \forall x, y \in E .
$$

With the aid of Bregman distance, we introduce the notion of the Bregman order regularity, and develop the approximation theory, the fixed point theorems results for the set-valued mappings for variational inequality problems in general Banach spaces.

Let $E$ be a Banach space whose (topological) dual we denote by $E^{*}$. Let $C$ be a nonempty, closed, and convex subset of $E$. Let $\Gamma: C \rightarrow E^{*}$ be a function. The variational inequality problem associated with $C$ and $\Gamma$ is denoted by $V I(C, \Gamma)$, and consists of finding an $\hat{x} \in C$ such that $\langle\Gamma(\hat{x}), x-\hat{x}\rangle \geq 0, \forall x \in C$. where $\Gamma: C \rightrightarrows E^{*}$ is any mapping, that is, $\Gamma$ is a function that maps $C$ into $2^{X^{*}} \backslash\{\emptyset\}$. the generalized variational inequality problem associated with $C$ and $\Gamma$ is to find an $\hat{x} \in C$ such that there exists a $\phi \in \Gamma(\hat{x})$ with

$$
\langle\phi, x-\hat{x}\rangle \geq 0, \quad \forall x \in C
$$

We refer to this problem succinctly as $\operatorname{GVI}(C, \Gamma)$. In turn, any $\hat{x} \in C$ such that (1.3) holds for some $\hat{y} \in \Gamma(\hat{x})$ is called a solution to $\operatorname{GVI}(C, \Gamma)$. If there is at least one solution to it, we say that $\operatorname{GVI}(C, \Gamma)$ is solvable. The first existence theorem for problem (1.3) was established by Hartman and Stampacchia in 1966 (see [5]). Since the celebrated existence theorem of Hartman and Stampacchia was obtained in 1966 (see [5]), the solvability of (generalized) variational inequalities has been studied by many authors, especially, in the context where $E$ is a Hilbert space (so that $E^{*}$ can be taken as $E$ ). It was proved that the solutions to $\mathrm{GVI}(\mathrm{C}, \Gamma)$ coincide with the fixed points of the self-mapping $P_{C} \circ\left(I_{E}-\Gamma\right)$, where $P_{C}$ is the metric projection operator onto $C$ in a Hilbert space $H$. Unfortunately, this observation fails when the norm of $E$ is not induced by an inner product. However, partly motivated by this, Alber [6] introduced the notion of the generalized metric projection operator $\pi_{C}: E^{*} \rightarrow C$ in a uniformly convex and uniformly smooth $E$. Noted that $\pi_{C}$ can be used instead of $P_{C}$ to convert a given solvability problem to a fixed point problem in a uniformly convex and uniformly smooth Banach spaces. To see more results on this subject, we refer to $[7,8,10,9,11]$ and the references therein.

The theory of fixed points and variational inequality problems with the set-valued mappings have been studied in the last forty years by using iterative methods; see, e.g., [12, 13, 14, 15]. Although there has been a large volume of literatures on the fixed point problem of nonlinear mappings and variational inequality problems, most of them are geared towards exploiting the symmetry property or the triangle inequality of metrics or using a seminorm (weak-norm) $p$ or a norm $\|\cdot\|$ on a vector space $X$ (see, e.g., $[16,17,18,19,20,21,22,23]$ ).

To the best of our knowledge, there is no study on the existence of variational inequality problems via the Bregman distances and Bregman functions in general Banach spaces. Our main focus in this paper is the existence of optimal solutions to the generalized variational inequality (1.3) of a nonself-mapping $\Gamma: C \rightrightarrows E^{*}$ with the aid of the Bregman distance induced by a strictly convex function $g: E \rightarrow \mathbb{R}$ in a reflexive Banach space $E$. Our results improve and generalize many known results; see, e.g., $[7,16,17,24]$ and the references therein. 


\section{Preliminaries}

In this section, we begin by recalling some facts and definitions, which will be used in the sequel.

2.1. Gradient and Bregman distances. Let $E$ be a Banach space. For any function $g: E \rightarrow$ $(-\infty,+\infty]$, the domain of $g$ is defined by

$$
\operatorname{dom} g=\{x \in E: g(x)<+\infty\} \text {. }
$$

$g$ is said to be proper if the interior of its domain is nonempty. We call a function $g: E \rightarrow \mathbb{R}$ lower semicontinuous if $\{x \in E: g(x) \leq r\}$ is closed for all $r$ in $\mathbb{R}$. Let $g: E \rightarrow \mathbb{R}$ be a proper (i.e., $g$ is not constantly $+\infty$ on $E$ ), lower semicontinuous, and strictly convex function. Let $x \in$ int dom $g$ and $y \in E$. The right-hand derivative of $g$ at $x$ in the direction $y$ is defined and denoted by

$$
g^{o}(x, y)=\lim _{t \downarrow 0} \frac{g(x+t y)-g(x)}{t} .
$$

The function $g$ is said to be Gâteaux differentiable at $x$ if $\lim _{t \rightarrow 0} \frac{g(x+t y)-g(x)}{t}$ exists for any $y$. In this case, $g^{o}(x, y)$ coincides with $\nabla g(x)$, the value of the gradient $\nabla g$ of $g$ at $x$. We call $g$ Fréchet differentiable at $x$ (see, e.g., [25, p. 13] or [26, p. 508]) if, for all $\varepsilon>0$, there exists $\delta>0$ such that

$$
|g(y)-g(x)-\langle y-x, \nabla g(x)\rangle| \leq \varepsilon\|y-x\| \quad \text { whenever }\|y-x\| \leq \delta .
$$

The function $g$ is said to be Fréchet differentiable if it is Fréchet differentiable everywhere. It is well known that if a continuous convex function $g: E \rightarrow \mathbb{R}$ is Gâteaux differentiable, then $\nabla g$ is norm-to-weak* continuous (see, e.g., [25, Proposition 1.1.10]). If $g$ is also Fréchet differentiable, then $\nabla g$ is norm-to-norm continuous (see [26, p. 508]).

For any $r>0$, let $B_{r}:=\{z \in E:\|z\| \leq r\}$. A function $g: E \rightarrow \mathbb{R}$ is said to be

- strongly coercive if

$$
\lim _{\left\|x_{n}\right\| \rightarrow+\infty} \frac{g\left(x_{n}\right)}{\left\|x_{n}\right\|}=+\infty
$$

- locally bounded if $g\left(B_{r}\right)$ is bounded for all $r>0$;

- locally uniformly convex on $E$ (or uniformly convex on bounded subsets of $E$ ([27, pp. $203,221])$ ) if the gauge $\rho_{r}:[0,+\infty) \rightarrow[0,+\infty]$ of uniform convexity of $g$, defined by

$$
\rho_{r}(t)=\inf _{x, y \in B_{r},\|x-y\|=t, \alpha \in(0,1)} \frac{\alpha g(x)+(1-\alpha) g(y)-g(\alpha x+(1-\alpha) y)}{\alpha(1-\alpha)},
$$

satisfies

$$
\rho_{r}(t)>0, \quad \forall r, t>0
$$

- locally uniformly smooth on $E$ ([27, pp. 207, 221]) if the function $\sigma_{r}:[0,+\infty) \rightarrow$ $[0,+\infty]$, defined by

$$
\sigma_{r}(t)=\sup _{x \in B_{r}, y \in S_{E}, \alpha \in(0,1)} \frac{\alpha g(x+(1-\alpha) t y)+(1-\alpha) g(x-\alpha t y)-g(x)}{\alpha(1-\alpha)},
$$


satisfies

$$
\lim _{t \downarrow 0} \frac{\sigma_{r}(t)}{t}=0, \quad \forall r>0 .
$$

Remark 2.1. For a locally uniformly convex map $g: E \rightarrow \mathbb{R}$, we have

$$
g(\alpha x+(1-\alpha) y) \leq \alpha g(x)+(1-\alpha) g(y)-\alpha(1-\alpha) \rho_{r}(\|x-y\|),
$$

for all $x, y$ in $B_{r}$ and for all $\alpha$ in $(0,1)$.

Lemma 2.1. [28, 29] Let $E$ be a Banach space, and let $g: E \rightarrow \mathbb{R}$ be a Gâteaux differentiable function, which is locally uniformly convex on E. Let $\left\{x_{n}\right\}_{n \in \mathbb{N}}$ and $\left\{y_{n}\right\}_{n \in \mathbb{N}}$ be bounded sequences in $E$. Then the following assertions are equivalent.

(1) $\lim _{n \rightarrow \infty} D_{g}\left(x_{n}, y_{n}\right)=0$.

(2) $\lim _{n \rightarrow \infty}\left\|x_{n}-y_{n}\right\|=0$.

For a proper lower semicontinuous convex function $g: E \rightarrow \mathbb{R}$, the subdifferential $\partial g$ of $g$ is defined by

$$
\partial g(x)=\left\{x^{*} \in E^{*}: g(x)+\left\langle y-x, x^{*}\right\rangle \leq g(y), \quad \forall y \in E\right\}
$$

for all $x$ in $E$. It is well known that $\partial g \subset E \times E^{*}$ is maximal monotone [30]. For any proper lower semicontinuous convex function $g: E \rightarrow \mathbb{R}$, the conjugate function $g^{*}$ of $g$ is defined by

$$
g^{*}\left(x^{*}\right)=\sup _{x \in E}\left\{\left\langle x, x^{*}\right\rangle-g(x)\right\}, \quad \forall x^{*} \in E^{*} .
$$

It is well known that

$$
g(x)+g^{*}\left(x^{*}\right) \geq\left\langle x, x^{*}\right\rangle, \quad \forall\left(x, x^{*}\right) \in E \times E^{*},
$$

and

$$
\left(x, x^{*}\right) \in \partial g \quad \text { is equivalent to } g(x)+g^{*}\left(x^{*}\right)=\left\langle x, x^{*}\right\rangle .
$$

It is worth mentioning that if $E$ is a reflexive Banach space, and $g: E \rightarrow \mathbb{R}$ is a continuous, convex, and Gâteaux differentiable function on $E$ then we have from [31] that

$$
g(x)+g^{*}(\nabla g(x))=\langle x, \nabla g(x)\rangle .
$$

We also know that if $g: E \rightarrow \mathbb{R}$ is a proper, lower semicontinuous, and convex function, then $g^{*}: E^{*} \rightarrow \mathbb{R}$ is a proper, weak* lower semicontinuous, and convex function.

Definition 2.1. [26] Let $E$ be a Banach space. The function $g: E \rightarrow \mathbb{R}$ is said to be a Bregman function if the following conditions are satisfied:

(1) $g$ is continuous, strictly convex, and Gâteaux differentiable;

(2) the set $\left\{y \in E: D_{g}(x, y) \leq r\right\}$ is bounded for all $x$ in $E$ and $r>0$.

Lemma 2.2. $[25,27]$ Let $E$ be a reflexive Banach space, and let $g: E \rightarrow \mathbb{R}$ be a strongly coercive Bregman function. Then

(1) $\nabla g: E \rightarrow E^{*}$ is one-to-one, onto, and norm-to-weak ${ }^{*}$ continuous;

(2) $\langle x-y, \nabla g(x)-\nabla g(y)\rangle=0$ if and only if $x=y$;

(3) $\left\{x \in E: D_{g}(x, y) \leq r\right\}$ is bounded for all $y$ in $E$ and $r>0$;

(4) dom $g^{*}=E^{*}, g^{*}$ is Gâteaux differentiable and $\nabla g^{*}=(\nabla g)^{-1}$. 
Lemma 2.3. (see [32]) Let $E$ be a Banach space, and let $C$ be a nonempty and convex subset of $E$. Let $g: E \rightarrow \mathbb{R}$ be a convex and Gâteaux differentiable function. Then, for $x \in E$ and $x_{0} \in C$, $D_{g}\left(x_{0}, x\right)=\min _{y \in C} D_{g}(y, x)$ if and only if $\left\langle y-x_{0}, \nabla g(x)-\nabla g\left(x_{0}\right)\right\rangle \leq 0, \forall y \in C$. Further, if $C$ is a nonempty, closed, and convex subset of a reflexive Banach space $E$, and $g: E \rightarrow \mathbb{R}$ is a strongly coercive Bregman function, then, for each $x \in E$, there exists a unique $x_{0} \in Q$ such that

$$
D_{g}\left(x_{0}, x\right)=\min _{y \in C} D_{g}(y, x) .
$$

The Bregman projection $P_{C}^{g}$ from $E$ onto $C$ is defined by $P_{C}^{g}(x)=x_{0}$ for all $x \in E$. It is also well known that $P_{C}^{g}$ has the following property:

$$
D_{g}\left(y, P_{C}^{g} x\right)+D_{g}\left(P_{C}^{g} x, x\right) \leq D_{g}(y, x)
$$

for all $y \in C$ and $x \in E$ (see [25] for more details).

2.2. Generalized metric projection operators. There are various ways of extending the classical metric projection operator from Hilbert spaces to Banach spaces. Let $E$ be a Banach space. Denote the operator norm on the dual $E^{*}$ by $\|\cdot\|_{*}$, and consider the map $V: E^{*} \times E \rightarrow \mathbb{R}$ defined by

$$
V(\psi, x):=\|\psi\|_{*}^{2}-2\langle\psi, x\rangle+\|x\|^{2} .
$$

(As $V(\psi, x) \geq\left(\|\psi\|_{*}-\|x\|\right)^{2}$ for all $\varphi$ and $x$, this map is nonnegative-valued, see Alber [6] and $\mathrm{Li}[17]$.

For any nonempty, closed, and convex subset $C$ of $E$, the Bregman generalized projection operator onto $C$ is the mapping $\Pi_{C}^{g}: E^{*} \rightrightarrows C$ defined by

$$
\Pi_{C}^{g}(\psi):=\left\{z \in C: W_{g}(z, \psi) \leq W_{g}(x, \psi) \text { for all } x \in C\right\} .
$$

If $E$ is a Hilbert space, then we can regard any $\psi$ in $E^{*}$ as lying in $E$ through the Riesz representation theorem. So, in that case, we have $W_{g}(x, \psi)=\|x-\psi\|^{2}$ for each $(\psi, x) \in E^{*} \times E$, which, in turn, implies that $\Pi_{C}^{g}$ is the standard metric projection operator onto $C$. More generally, provided that $E$ is reflexive, $\Pi_{C}^{g}$ has nonempty, closed, bounded, and convex values, and it is single-valued if and only if $E$ is strictly convex. For these results, and other important properties of $\Pi_{C}^{g}$, we refer to $[14,33]$.

2.3. Variational characterization of $\Pi_{C}^{g}$. The variational characterization of the metric projection operator in the context of Hilbert spaces plays an important role in studying variational inequalities. A similar characterization also holds for the generalized metric projection operator in certain cases. To introduce this characterization, we now state the variational characterization of Bregman generalized projection operators. If $E$ is a reflexive Banach space, and $C$ is a nonempty, closed, and convex subset of $E$, then, for any $\psi \in E^{*}$,

$$
x \in \Pi_{C}^{g}(\psi) \Longleftrightarrow\langle x-y, \psi-\nabla g(x)\rangle \geq 0 \text { for every } y \in C .
$$

(For the proof, see Li [17].) A standard argument based on this characterization yields the main connection between fixed point problems and variational inequalities.

Theorem 2.1. Let $E$ be a reflexive Banach space, and let $\lambda: E \rightarrow(0,+\infty)$ be a function. Let $C$ be a nonempty, closed, and convex subset of $E$, and let $\Gamma: C \rightrightarrows E^{*}$ be a set-valued mapping. 
Then, $\hat{x}$ is a solution to $G V I(C, \Gamma)$ if and only if $\hat{x}$ is a fixed point of the mapping $\Pi_{C}^{g} \circ(\nabla g-\lambda \Gamma)$, that is,

$$
\hat{x} \in \Pi_{C}^{g}(\nabla g(\hat{x})-\lambda(\hat{x}) \Gamma(\hat{x})) .
$$

Proof. Suppose that $\hat{x} \in C$ is a fixed point of $\Pi_{C}^{g} \circ(\nabla g-\lambda \Gamma)$, that is, there exists $\hat{\psi} \in \Gamma(\hat{x})$ such that

$$
\hat{x} \in \Pi_{C}^{g}(\nabla g(\hat{x})-\lambda(\hat{x}) \hat{\psi}) .
$$

In view of Lemma 2.3, (2.2), and (3.2), we conclude that

$$
\begin{aligned}
W_{g}(\hat{x}, \nabla g(\hat{x})-\lambda(\hat{x}) \hat{\psi}) & =\inf _{y \in C} W_{g}(y, \nabla g(\hat{x})-\lambda(\hat{x}) \hat{\psi}) \\
& =\inf _{y \in C} D_{g}\left(y, \nabla g^{*}(\nabla g(\hat{x})-\lambda(\hat{x}) \hat{\psi})\right)
\end{aligned}
$$

Employing Lemma 2.3, we are led to $\hat{x} \in P_{C}^{g}\left(\nabla g^{*}(\nabla g(\hat{x})-\lambda(\hat{x}) \hat{\psi})\right.$. Hence, it follows from (2.1) that

$$
\left\langle y-\hat{x}, \nabla g\left(\nabla g^{*}(\nabla g(\hat{x})-\lambda(\hat{x}) \hat{\psi})\right)-\nabla g(\hat{x})\right\rangle \leq 0, \quad \forall y \in C
$$

This implies that

$$
\langle y-\hat{x},-\lambda(\hat{x}) \hat{\psi}\rangle=\left\langle y-\hat{x}, \nabla g\left(\nabla g^{*}(\nabla g(\hat{x})-\lambda(\hat{x}) \hat{\psi})-\nabla g(\hat{x})\right\rangle \leq 0, \quad \forall y \in C .\right.
$$

Hence $\langle y-\hat{x}, \lambda(\hat{x}) \hat{\psi}\rangle \geq 0, \forall y \in C$, which means that (2.3) is valid. The converse implication follows from reversing the steps of this argument.

2.4. Posets and lattices. Let $(X, \succcurlyeq)$ be a partially ordered set (poset), and let $S$ be a subset of $X$. An element $x$ of $X$ is called an $\succcurlyeq$-upper bound for $S$ if $x \succcurlyeq S$, that is, $x \succcurlyeq y$ for each $y \in S$. (The notation $S \succcurlyeq x$ is similarly understood.) We say that $S$ is $\succcurlyeq$-bounded from above if $x \succcurlyeq S$ for some $x \in X$, and $\succcurlyeq$-bounded from below if $S \succcurlyeq x$ for some $x \in X$. In turn, $S$ is said to be $\succcurlyeq$-bounded if it is $\succcurlyeq$-bounded both from above and below. As usual, we say that a sequence $\left\{x_{m}\right\}_{m \in \mathbb{N}}$ in $X$ is said to be $\succcurlyeq$-bounded (from above/below) if $\left\{x_{1}, x_{2}, \ldots\right\}$ possesses this property. If $x \in S$ and $x$ is an $\succcurlyeq$-upper bound for $S$, we say that $x$ is the $\succcurlyeq$-maximum in $S$. (The $\succcurlyeq$-minimum element of $S$ is similarly defined.) A nonempty subset $S$ of $X$ is said to be a $\succcurlyeq$-chain in $E$ if either $x \succcurlyeq y$ or $y \succcurlyeq x$ hold for each $x, y \in X$.

The $\succcurlyeq$-supremum of $S$ is the $\succcurlyeq$-minimum of the set of all $\succcurlyeq$-upper bounds for $S$, and is denoted by $\bigvee_{X} S$. (The $\succcurlyeq$-infimum of $S$ - denoted as $\bigwedge_{X} S$ - is defined as the $\preccurlyeq$-supremum of $S$.) As conventional, we denote $\bigvee_{X}\{x, y\}$ as $x \vee y$, and $\bigwedge_{X}\{x, y\}$ as $x \wedge y$, for any $x, y \in E$. If $x \vee y$ and $x \wedge y$ exist for every $x$ and $y$ in $X$, we say that $(E, \succcurlyeq)$ is a lattice. Ff $\bigvee_{X} S$ and $\bigwedge_{X} S$ exist for every nonempty ( $\succcurlyeq$-bounded) $S \subseteq X$, we say that $(X, \succcurlyeq)$ is a (Dedekind) complete lattice. Finally, if $Y$ is a nonempty subset of $X$, which contains $\bigvee_{X}\{x, y\}$ and $\bigwedge_{X}\{x, y\}$ for every $x, y \in X$, then it is said to be a $\succcurlyeq$-sublattice of $X$. In turn, if $Y$ contains $\vee_{X} S$ and $\wedge_{X} S$ for every nonempty $S \subseteq Y$, then it is said to be a subcomplete $\succcurlyeq$-sublattice of $X$. (Easy examples show that a $\succcurlyeq$-sublattice of $X$, which happens to be a complete lattice with respect to $\succcurlyeq$ need not be a subcomplete $\succcurlyeq$-sublattice of $X$.) 
2.5. Order-preservation for mappings. Let $\left(X, \succcurlyeq_{X}\right)$ and $\left(Y, \succcurlyeq_{Y}\right)$ be two lattices. A map $F$ : $X \rightarrow Y$ is said to be order-preserving if

$$
x \succcurlyeq_{X} y \Longrightarrow F(x) \succcurlyeq_{Y} F(y)
$$

for every $x, y \in X$. In turn, if $\Gamma: X \rightrightarrows Y$ is a mapping- by this we mean that $\Gamma$ is a map from $X$ into $2^{Y} \backslash\{\emptyset\}$. We say that $\Gamma$ is upper order-preserving if $x \succcurlyeq_{X} y$ implies that for every $y \in \Gamma(y)$ there is an $x \in \Gamma(x)$ such that $x \succcurlyeq_{Y} y$. (It is easily checked that for any such $\Gamma$ the map $x \mapsto \bigvee_{Y} \Gamma(x)$ is order-preserving, provided that it is well-defined.) Similarly, $\Gamma$ is lower orderpreserving if $x \succcurlyeq_{X} y$ implies that, for every $x \in \Gamma(x)$, there is a $y \in \Gamma(y)$ such that $x \succcurlyeq_{Y} y$. (For any such $\Gamma$, the map $x \mapsto \bigwedge_{Y} \Gamma(x)$ is order-preserving provided that it is well-defined.) Finally, $\Gamma$ is said to be order-preserving if it is both upper and lower order-preserving. (If $\left(X, \succcurlyeq_{X}\right)$ and $\left(Y, \succcurlyeq_{Y}\right)$ are sublattices of a given lattice $(Z, \succcurlyeq)$, then we use the phrase $\succcurlyeq$-preserving instead of order-preserving.) We say that $\Gamma$ has topped (bottomed) values if there is a $\succcurlyeq_{Y}$-maximum ( $\succcurlyeq_{Y^{-}}$ minimum) in $\Gamma(x)$ for each $x \in X$. A Riesz space is a lattice $(X, \succcurlyeq)$, where $X$ is a (real) linear space whose linear structure is compatible with the partial order $\succcurlyeq$ in the sense that $\alpha i d_{X}+z$ is a $\succcurlyeq$-preserving self-map on $X$ for every $z \in X$ and real number $\alpha>0$. The positive cone of $(X, \succcurlyeq)$ is $X_{+}:=\{x \in X: x \succcurlyeq 0\}$, which is a (pointed) convex cone in $X$. A Riesz space $(X, \succcurlyeq)$ is called a normed Riesz space if $X$ is a normed linear space whose norm $\|\cdot\|$ is compatible with the partial order $\succcurlyeq$ in the sense that $\|x\| \geq\|y\|$ holds for every $x, y \in X$ with $|x| \succcurlyeq|y|$. If $(X, \succcurlyeq)$ is a normed Riesz space, it is readily checked that the lattice operations $\vee$ and $\wedge$ are continuous maps from $X \times X$ into $X$. As an immediate consequence of this fact, we find that $X_{+}$ is a closed cone in $E$. (As $X_{+}$is convex, it is weakly closed as well.) In turn, this implies that $x_{m} \rightarrow \bigvee_{X}\left\{x_{1}, x_{2}, \ldots\right\}$ for every convergent sequence $\left\{x_{m}\right\}_{m \in \mathbb{N}}$ in $E$ with $\cdots \succcurlyeq x_{2} \succcurlyeq x_{1}$.

2.6. Order-Dual. Let $(E, \succcurlyeq)$ be a Banach lattice. The dual of $\succcurlyeq$ is the partial order $\succcurlyeq^{*}$ on $E^{*}$ defined as follows:

$$
\psi \succcurlyeq^{*} \phi \Longleftrightarrow\langle x, \psi-\phi\rangle \geq 0 \text { for every } x \in E_{+} .
$$

It is well known that $\left(E^{*}, \succcurlyeq^{*}\right)$ is a Banach lattice, which is called the dual of $(E, \succcurlyeq)$. As usual, we denote the positive cone of $\left(E^{*}, \succcurlyeq^{*}\right)$ by $E_{+}^{*}$, and recall that $x \in E_{+}$if and only if $\langle x, \psi\rangle \geq 0$ for every $\psi \in E_{+}^{*}$. (This is easily proved by using the Hahn-Banach theorem; see Meyer-Nieberg [34, Proposition 1.4.2]).

\section{BREGMAN ORDER-REGULARITY}

3.1. Bregman order-regular Banach lattices. In the context of Hilbert lattices, Isac [20] shown that the metric projection onto any nonempty, closed, and convex sublattice is sure to be order-preserving, and this plays a key role for the order-theoretic analysis of variational inequalities. Unfortunately, this property may not hold for generalized projection operator in the context of even finite-dimensional Banach lattices; see, e.g., [16].

We now introduce a sufficient condition for the sublattices $C$ of a reflexive Banach lattice $E$ that would ensure the order-preservation of the Bregman generalized projection operator onto $C$. Let $(E, \succcurlyeq)$ be a Banach lattice. A $\succcurlyeq$-sublattice $Y$ of $E$ is said to be Bregman regular if $g($.$) is$ submodular on $Y$ with respect to $\succcurlyeq$, that is,

$$
g(x \vee y)+g(x \wedge y) \leq g(x)+g(y) \quad \text { for every } x, y \in Y .
$$


Obviously, if $(E, \succcurlyeq)$ is itself Bregman regular, then every $\succcurlyeq$-sublattice of $E$ is Bregman regular. To see some facts and examples about the order-regularity and order-regular sublattices of Banach lattices, we refer the reader to [16].

3.2. Bregman generalized projections onto Bregman regular sublattices. We mention that the Bregman regularity of a sublattice of a reflexive Banach lattice guarantees the order-preservation of the Bregman generalized projection operator onto this sublattice. The following result is the aim of this topic.

Proposition 3.1. Let $(E, \succcurlyeq)$ be a reflexive Banach lattice, and let $C$ be a Bregman regular $\succcurlyeq-$ sublattice of $E$. Let $g: E \rightarrow \mathbb{R}$ be a Gâteaux differentiable function, which is locally uniformly convex on $E$. Then, $\Pi_{C}^{g}$ is order-preserving.

Proof. Assume, on the contrary, that $\Pi_{C}^{g}$ is not upper order-preserving. Then, there exist $\psi$ and $\phi$ in $E^{*}$ such that (i) $\psi \succcurlyeq^{*} \phi$; and (ii) there exists $y \in \Pi_{C}^{g}(\phi)$ such that $z \succcurlyeq y$ is not valid for any $z \in \Pi_{C}^{g}(\psi)$. Let $x$ in $\Pi_{C}^{g}(\psi)$ be arbitrarily chosen. If we set $z:=x \vee y$ in (ii), then we deduce that $x \vee y$ does not belong to $\Pi_{C}^{g}(\psi)$ while $x \vee y \in C$ due to the fact that $C$ is a sublattice of $E$. It follows from the definition of $\Pi_{C}^{g}$ that $W_{g}(x, \psi)<W_{g}(x \vee y, \psi)$, that is,

$$
\langle x \vee y-x, \psi\rangle<g(x \vee y)-g(x) \text {. }
$$

On the other hand, as $x \wedge y \in C$ (because $C$ is a sublattice of $E$ ), we deduce that $W_{g}(y, \phi) \leq$ $W_{g}(x \wedge y, \phi)$, that is, $\langle x \wedge y-y, \phi\rangle \leq g(x \wedge y)-g(y)$. Employing the relation $x \vee y+x \wedge y=x+y$, we can express this inequality as

$$
\langle x \vee y-x,-\phi\rangle \leq g(x \wedge y)-g(y)
$$

Combining (3.1) and (3.2) amounts to

$$
\langle x \vee y-x, \psi-\phi\rangle<g(x \vee y)+g(x \wedge y)-(g(x)+g(y))
$$

Thus, by Bregman $\succcurlyeq$-regularity of $C$, we obtain $\langle x \vee y-x, \psi-\phi\rangle<0$. In view of (i), since $\psi-\phi \in E_{+}^{*}$, by the discussion in Section 2.5 we deduce that $x \vee y-x$ does not belong to $E_{+}$, that is, $x \vee y \succcurlyeq x$ is false. This is a contradiction. This proves that $\Pi_{C}^{g}$ is upper order-preserving. By the similar arguments, one can prove the lower order-preservation. This completes the proof.

\section{Optimal SolvabiLity of VARIATIONAL INEQUALITIES}

4.1. Variational inequalities on bounded sublattices. In this section, we employ the following well-known result whose proof is based on theorder-theoretic fixed point theory. The following result is a special case of the Abian-Brown fixed point theorem; see [34].

Theorem 4.1. Let $(X, \succcurlyeq)$ be a complete lattice, and let $f: X \rightarrow X$ be a $\succcurlyeq$-preserving self-map on $X$. Then, for any $x_{*} \in X$ with $f\left(x_{*}\right) \succcurlyeq x_{*}$, there is a fixed point $x$ of $f$ with $x \succcurlyeq x_{*}$.

Our first main result identifies certain types of generalized variational inequalities on the subcomplete sublattices of a suitable Banach lattice.

Theorem 4.2. Let $(E, \succcurlyeq)$ be a reflexive Banach lattice, and let $C$ be a Bregman regular $\succcurlyeq-$ sublattice of $E$. Let $g: E \rightarrow \mathbb{R}$ be a Gâteaux differentiable function, which is locally uniformly convex on $E$. Let $C$ be a Bregman regular, closed, and convex subcomplete $\succcurlyeq$-sublattice of E. 
Let $\Gamma: C \rightrightarrows E^{*}$ be a mapping such that $\nabla g-\lambda \Gamma$ is upper (lower) order-preserving with topped (bottomed) values, for some $\lambda: C \rightarrow(0,+\infty)$. Then, there is a $\succcurlyeq$-maximum ( $\succcurlyeq$-minimum) solution to $G \operatorname{VI}(C, \Gamma)$.

Proof. We will only investigate the case that $\Gamma$ is upper order-preserving. For the argument of the lower order-preserving case, it can be processed analogously. For the simplicity notation, we set $\Theta:=\nabla g-\lambda \Gamma$, and note that the set of all fixed points of $\Pi_{C}^{g} \circ \Theta$ - we denote this set by $F i x\left(\Pi_{C}^{g} \circ \Theta\right)$ - is equal to the set of all solutions to $G V I(C, \Gamma)$ by Theorem 2.1. It suffices to show that $\operatorname{Fix}\left(\Pi_{C}^{g} \circ \Theta\right)$ is a nonempty set that has a $\succcurlyeq$-maximum. Let us define the map $\Psi: C \rightarrow E^{*}$ by

$$
\Psi(x):=\bigvee_{E^{*}} \Theta(x)
$$

Since the values of $\Theta$ are topped by hypothesis, $\Psi$ is well-defined. Let us verify that $\Psi$ is orderpreserving. To this end, take any $x$ and $y$ in $C$ with $x \succcurlyeq y$, and fix an arbitrary $\phi \in \Theta(y)$. Since $\Theta$ is upper order-preserving by hypothesis, there is a $\psi$ in $\Theta(x)$ such that $\psi \succcurlyeq^{*} \phi$. This entails that $\bigvee_{E^{*}} \Theta(x) \succcurlyeq^{*} \phi$. Thus, as $\phi$ is arbitrary in $\Theta(y)$, we conclude that $\Psi(x)$ is an upper $\succcurlyeq^{*}$-bound for $\Theta(y)$. Hence, $\Psi(x) \succcurlyeq^{*} \bigvee_{E^{*}} \Theta(y)=\Psi(y)$. Thus we have that $\Psi$ is order-preserving. This, together with Proposition 3.1, implies that $\Pi_{C}^{g} \circ \Psi$ is a $\succcurlyeq$-preserving self-map on $C$. By Theorem 4.1 (with $x^{*}=\bigwedge_{E} C$ ), we have that there exists a fixed point of $\Pi_{C}^{g} \circ \Psi$. But, Fix $\left(\Pi_{C}^{g} \circ \Psi\right) \subseteq$ $F i x\left(\Pi_{C}^{g} \circ \Theta\right)$. (If $x$ is a fixed point of $\Pi_{C}^{g} \circ \Psi$, then, because $\Theta(x)$ is topped, we have $\Psi(x) \in \Theta(x)$. Hence, $x=\Pi_{C}^{g}(\Psi(x)) \in \Pi_{C}^{g}(\Theta(x))$.) This implies that $F i x\left(\Pi_{C}^{g} \circ \Theta\right) \neq \emptyset$. To complete the proof, we must show that $\operatorname{Fix}\left(\Pi_{C}^{g} \circ \Theta\right)$ contains its $\succcurlyeq$-maximum. Now, we set $x_{*}:=\bigvee_{E} F i x\left(\Pi_{C}^{g} \circ \Theta\right)$, which belongs to $C$ (because $C$ is subcomplete). Since $\Pi_{C}^{g} \circ \Psi$ is $\succcurlyeq$-preserving, and $\Pi_{C}^{g}$ is order-preserving, the definition of $x_{*}$ amounts to

$$
\begin{aligned}
\Pi_{C}^{g}\left(\Psi\left(x_{*}\right)\right) & \succcurlyeq \Pi_{C}^{g}\left(\Psi\left(F i x\left(\Pi_{C}^{g} \circ \Theta\right)\right)\right) \\
& \succcurlyeq \bigvee_{E} \Pi_{C}^{g}\left(\Theta\left(F i x\left(\Pi_{C}^{g} \circ \Theta\right)\right)\right) \\
& \succcurlyeq \bigvee_{E} \operatorname{Fix}\left(\Pi_{C}^{g} \circ \Theta\right)
\end{aligned}
$$

where the last ordering is obtained by the inclusion $F i x\left(\Pi_{C}^{g} \circ \Theta\right) \subseteq\left(\Pi_{C}^{g} \circ \Theta\right)\left(F i x\left(\Pi_{C}^{g} \circ \Theta\right)\right)$. This amounts to $\Pi_{C}^{g}\left(\Psi\left(x_{*}\right)\right) \succcurlyeq x_{*}$. Given that $\Pi_{C}^{g} \circ \Psi$ is a $\succcurlyeq$-preserving self-map on $C$, therefore, we may apply Theorem 4.1 to conclude that there is a fixed point $x$ of $\Pi_{C}^{g} \circ \Psi$ such that $x \succcurlyeq x_{*}$. As $F i x\left(\Pi_{C}^{g} \circ \Psi\right) \subseteq F i x\left(\Pi_{C}^{g} \circ \Theta\right)$, we also have $x \in F i x\left(\Pi_{C}^{g} \circ \Theta\right)$. From the definition of $x_{*}$, we must have $x=x_{*}$. This implies that $x$ is a fixed point of $\Pi_{C}^{g} \circ \Theta$, that is, $F i x\left(\Pi_{C}^{g} \circ \Theta\right)$ contains its $\succcurlyeq$-maximum.

When $\Gamma$ is single-valued, we can improve the conclusion of Theorem 4.1 substantially, which extends Theorem 4.2 of Nishimura and Ok [11] to the context of Banach lattices.

Theorem 4.3. Let $(E, t)$ be a reflexive Banach lattice, and let $C$ be a Bregman regular, closed, and convex subcomplete $\succcurlyeq$ - sublattice of $E$. Let $g: E \rightarrow \mathbb{R}$ be a Gâteaux differentiable function, which is locally uniformly convex on $E$. Let $F: C \rightarrow E^{*}$ be a function such that $\nabla g-\lambda F$ is order-preserving for some $\lambda: C \rightarrow(0,+\infty)$. Then, the set of all solutions to $\operatorname{VI}(C, F)$ is a complete lattice relative to $\succcurlyeq$. 
Proof. By the same arguments used in the proof of Theorem 4.2 (with $F$ playing the role of $\Gamma$ ), we have $\Psi=\Theta$. Consequently, as shown in the second paragraph of that proof, $\Pi_{C}^{g} \circ \Psi$, that is, $\Pi_{C}^{g} \circ(\nabla g-\lambda F)$ is a $\succcurlyeq$-preserving self-map on $C$. In view of the Knaster-Tarski fixed point theorem, we deduce that $\left(\operatorname{Fix}\left(\Pi_{C}^{g} \circ(\nabla g-\lambda F)\right), \succcurlyeq\right)$ is a complete lattice. Employing Theorem 2.1, we conclude the desired conclusion, which completes the proof.

4.2. Variational inequalities on unbounded sublattices. In this subsection, we extend the solvability results of the previous selection to the case of generalized variational inequalities on sublattices that need not to be subcomplete.

Theorem 4.4. Let $(E, \succcurlyeq)$ be a reflexive Banach lattice, and let $C$ be a Bregman regular, closed, and convex $\succcurlyeq$-sublattice of $E$. Let $g: E \rightarrow \mathbb{R}$ be a Gâteaux differentiable function, which is locally uniformly convex on $E$. Let $\Gamma: C \rightrightarrows X^{*}$ be a mapping such that $\nabla g-\lambda \Gamma$ is upper orderpreserving with topped values, for some $\lambda: C \rightarrow(0,+\infty)$. If there exist $z_{\circ}, z^{\circ} \in C$ such that $\Gamma\left(z^{\circ}\right) \succcurlyeq^{*} 0 \succcurlyeq^{*} \Gamma\left(z_{\circ}\right)$, then $G V I(C, \Gamma)$ is solvable.

Proof. We set $D:=\left\{x \in C: z^{\circ} \succcurlyeq x \succcurlyeq z_{\circ}\right\}$ and $\Theta:=\nabla g-\lambda \Gamma$. It is our aim to show that $\Pi_{C}^{g} \circ \Theta(D) \subseteq D$. To see this, we first notice that $\Gamma\left(z^{\circ}\right) \succcurlyeq^{*} 0$ and $\lambda\left(z^{\circ}\right)>0$ imply $\nabla g\left(z^{\circ}\right) \succcurlyeq^{*}$ $\nabla g\left(z^{\circ}\right)-\lambda \Gamma\left(z^{\circ}\right)$. So, $\Pi_{C}^{g}\left(\nabla g\left(z^{\circ}\right)\right) \succcurlyeq \Pi_{C}^{g} \circ \Theta\left(z^{\circ}\right)$ thank to Proposition 3.1. From $\Pi_{C}^{g} \circ \nabla g=i d_{C}$ (Subsection 2.4), we also obtain that $z^{\circ} \succcurlyeq \Pi_{C}^{g}\left(\Theta\left(z^{\circ}\right)\right)$. Now, one takes any $x \in C$ so that $z^{\circ} \succcurlyeq x$. Since $\Pi_{C}^{g}$ is order-preserving and $\Theta$ is upper order-preserving, $\Pi_{C}^{g} \circ \Theta$ is upper order-preserving. Therefore, for any $y$ in $\Pi_{C}^{g}(\Theta(x))$, there is a $y^{\circ} \in \Pi_{C}^{g}\left(\Theta\left(z^{\circ}\right)\right)$ such that $y^{\circ} \succcurlyeq y$. It follows from $z^{\circ} \succcurlyeq \Pi_{C}^{g}\left(\Theta\left(z^{\circ}\right)\right)$ that $z^{\circ} \succcurlyeq y^{\circ} \succcurlyeq y$. Since $y$ is arbitrary in $\Pi_{C}^{g}(\Theta(x))$, it follows that $z^{\circ} \succcurlyeq \Pi_{C}^{g}(\Theta(x))$. By a similar argument, we can show that $\Pi_{C}^{g}(\Theta(x)) \succcurlyeq z_{\circ}$. Hence $\Pi_{C}^{g}(\Theta(x)) \in D$, which proves our claim. Since $C$ is a $\succcurlyeq$-sublattice of $E, D$ is a subcomplete $\succcurlyeq$-sublattice of $E$. Furthermore, $D=\left(z_{\circ}+E_{+}\right) \cap\left(z^{\circ}-E_{+}\right)$. Observe that $E_{+}$is closed and convex, so is $D$. Finally, $D$ is Bregman regular, due to $C$. Employing Theorem 4.1, we deduce that there is a solution, say, $\hat{x}$, to $G V I\left(C, \Gamma||_{D}\right)$. Then, in view of Theorem 2.1, $\hat{x} \in \Pi_{D}^{g} \circ(\nabla g-\lambda \Gamma)(\hat{x}) \subseteq D$. This implies that there is a $\hat{\phi} \in \Theta(\hat{x})$ such that $\hat{x}=\Pi_{D}^{g}(\hat{\phi})$. By what we have shown in the previous paragraph, $\Pi_{C}^{g}(\hat{\phi}) \in D$. Thus, by definition of $\Pi_{C}^{g}$, we have $W_{g}\left(\hat{\phi}, \Pi_{D}^{g}(\hat{\phi})\right) \leq W_{g}\left(\hat{\phi}, \Pi_{C}^{g}(\hat{\phi})\right)$. But, as $\Pi_{D}^{g}(\hat{\phi}) \in D \subseteq C$ and $\Pi_{C}^{g}$ is single-valued, this is possible if and only if $\Pi_{D}^{g}(\hat{\phi})=\Pi_{C}^{g}(\hat{\phi})$. Thus, $\hat{x}=\Pi_{C}^{g}(\hat{\phi})$, which yields that $\hat{x}$ is a fixed point of $\Pi_{C}^{g} \circ \Theta$, and hence a solution to $G V I(D, \Gamma)$. This completes the proof.

In order to guarantee the existence of an optimal solution in the context of Theorem 4.2, we need to assume that $\Gamma$ "condenses" its domain in an order-theoretic sense. Based on this, we have the following result.

Theorem 4.5. Let $(E, t)$ be a reflexive Banach lattice, and let $C$ be a Bregman regular, closed, and convex $\succcurlyeq$-sublattice of $E$. Let $g: E \rightarrow \mathbb{R}$ be a strictly convex and Gateaux differentiable function. Let $\Gamma: C \rightrightarrows E^{*}$ be a mapping such that $\nabla g-\lambda \Gamma$ is upper (lower) order-preserving with topped (bottomed) values, for some $\lambda: C \rightarrow(0,+\infty)$. If $(\nabla g-\lambda \Gamma)(C)$ contains its $\succcurlyeq *$ maximum and $\succcurlyeq^{*}$-minimum, then there is a $\succcurlyeq$-maximum ( $\succcurlyeq-$ minimum) solution to $G V I(C, \Gamma)$.

Proof. We set $\Theta:=\nabla g-\lambda \Gamma$ again, and denote the $\succcurlyeq^{*}$-maximum and $t^{*}$-minimum of $\Theta(C)$ by $\psi^{\circ}$ and $\psi_{\circ}$, respectively. By Proposition 3.1, we have

$$
\Pi_{C}^{g}\left(\psi^{\circ}\right) \succcurlyeq \Pi_{C}^{g}(\Theta(C)) \succcurlyeq \Pi_{C}^{g}\left(\psi_{\circ}\right)
$$


Thus $D:=\left\{x \in C: \Pi_{C}^{g}\left(\psi^{\circ}\right) \succcurlyeq x \succcurlyeq \Pi_{C}^{g}\left(\psi_{\circ}\right)\right\}$ is nonempty. This set is an order-interval, so it is a closed and convex subcomplete $\succcurlyeq$-sublattice of $C$ (as in the proof of the previous result). We claim that

$$
\operatorname{Fix}\left(\Pi_{C}^{g} \circ \Theta\right)=F i x\left(\left.\Pi_{D}^{g} \circ \Theta\right|_{D}\right) .
$$

Indeed, if $x \in F i x\left(\Pi_{C}^{g} \circ \Theta\right)$, then $x=\Pi_{C}^{g}(\psi)$ for some $\psi \in \Theta(x)$. As $x \in C$, (4.2) entails that $x=$ $\Pi_{C}^{g}(\psi) \in C$, so $W_{g}\left(\psi, \Pi_{D}^{g}(\psi)\right) \leq W_{g}(\psi, x)$. On the other hand, as $x=\Pi_{C}^{g}(\psi)$ and $\Pi_{C}^{g}(\psi) \in D \subseteq$ $C$, this is possible if and only if $x=\Pi_{C}^{g}(\psi)$ because $\Pi_{C}^{g}$ is single-valued. Thus, $x \in \Pi_{D}^{g}(\Theta(x))$, that is, $x$ is a fixed point of $\left.\Pi_{D}^{g} \circ \Theta\right|_{D}$. Conversely, if $x \in F i x\left(\left.\Pi_{C}^{g} \circ \Theta\right|_{C}\right)$, then $x=\Pi_{D}^{g}(\psi)$ for some $\psi \in \Theta(x)$. As $x \in D \subseteq C$, (4.1) ensures that $\Pi_{C}^{g}(\psi) \in C$, so $W_{g}(x, \psi) \leq W_{g}\left(\Pi_{C}^{g}(\psi), \psi\right)$ by definition of $\Pi_{D}^{g}$. Since $x \in C$, this can happen if and only if $x=\Pi_{C}^{g}(\psi)$, which implies $x=\Pi_{C}^{g}(\psi) \in \Pi_{C}^{g}(\Theta(x))$. Employing Theorems 2.1 and 4.1 to $G V I\left(C,\left.\Gamma\right|_{D}\right)$ and using (4.2), we arrive at the desired conclusion, which completes the proof.

\section{Order-Theoretic FiXed PoInt Theory}

In this section, we apply our main results in the setting of fixed point theory. Let $E$ be a Banach space, $S$ a nonempty subset of $E$, and $\Gamma: S \rightrightarrows E^{*}$ any set-valued mapping. We say that a point $x$ in $S$ as a Bregman fixed point of $\Gamma$ if $\nabla g(x) \in \Gamma(x)$. It is evident that, when $E=H$ is a Hilbert space, the notion of Bregman fixed points reduce to that of fixed points. There are various fixed point theorems, such as the Browder-Göhde-Kirk fixed point theorem, about functions that map a closed and convex subset $C$ of a Hilbert space $H$ into $H$ such that the line segment between $x$ and $T(x)$ contains at least two points in $C$ so long as $x \neq T(x)$. In the following, we present an order-theoretic formulation of this type of a fixed point theorem as an application of our main theorems in the context of a Banach space.

Theorem 5.1. Let $(E, \succcurlyeq)$ be a reflexive Banach lattice, and let $C$ be a Bregman regular, closed, and convex subcomplete $\succcurlyeq$-sublattice of $E$. Let $g: E \rightarrow \mathbb{R}$ be a strictly convex and Gateaux differentiable function. Let $\Gamma: C \rightrightarrows E^{*}$ be an upper order-preserving mapping with topped values such that

$$
|\operatorname{co}\{x, y\} \cap C| \geq 2 \text { for all }(x, y) \in C \times E \text { with } \nabla g(x) \neq \nabla g(y) \in \Gamma(x) .
$$

Then, $\Gamma$ has a dual fixed point.

Proof. Setting $\Phi:=\nabla g-\Gamma$, we deduce that $\nabla g-\Phi$ is an upper order-preserving mapping from $C$ into $E^{*}$ with topped values. In view of Theorem 4.1, there is an $x \in C$ and $\psi \in \Gamma(x)$ such that $\left\langle z-x, \psi_{x}-\psi\right\rangle \geq 0$ for every $z \in C$, where $\psi_{x}:=\nabla g(x)$. It is our aim to show that $\psi=\psi_{x}$. Assume on the contrary that $\psi \neq \psi_{x}$. Then, by reflexivity of $E, \nabla g$ is surjective, which implies that $\psi=\psi_{y}$ for some $y \in E$. As $\psi \neq \psi_{x}$, we may employ (5.1) to find a $\lambda \in(0,1]$ such that $x-\lambda(x-y)=(1-\lambda) x+\lambda y \in C$. Hence $\left\langle x-\lambda(x-y)-x, \psi_{x}-\psi_{y}\right\rangle \geq 0$ that is, $\left\langle x-y, \psi_{x}-\psi_{y}\right\rangle \leq 0$.

On the other hand, in view of (1.2), we conclude that

$$
0 \leq D_{g}(x, y)=-D_{g}(y, x)+\left\langle x-y, \psi_{x}-\psi_{y}\right\rangle \leq\left\langle x-y, \psi_{x}-\psi_{y}\right\rangle \leq 0,
$$

which yields that $\left\langle x-y, \psi_{x}-\psi_{y}\right\rangle=0$. In view of Lemma 2.1, we deduce that $\psi_{x}=\psi_{y}$, a contradiction. Thus we obtain $\nabla g(x)=\psi_{x}=\psi \in \Gamma(x)$, which completes the proof. 
Remark 5.1. The technique of using a strictly convex function $g: E \rightarrow \mathbb{R}$ produces a Bregman distance that can be considered as the generality of our method. It is worth mentioning that, for the special case of $g(x)=\|x\|^{2}$ for all $x$ in $E$, we obtain that $\nabla g(x)=2 J x$ for all $x$ in $E$, where $J$ is the normalized duality mapping from $E$ into $E^{*}$. The main advantage of the main results is that by using a Bregman function $g: E \rightarrow \mathbb{R}$, we are led to the order-preserving property of the Bregman generlized projection $\Pi_{C}^{g}$, which yields the existence of optimal solutions of variational inequlity (1.3).

\section{Acknowledgments}

The author would like to thank the editor and the referees for valuable evaluation and constructive comments which improved the paper considerably.

\section{REFERENCES}

[1] W. Takahashi, Nonlinear Functional Analysis, Fixed Point Theory and its Applications, Yokahama Publishers, Yokahama, 2000.

[2] L. M. Bregman, The relation method of finding the common point of convex sets and its application to the solution of problems in convex programming, USSR Comput. Math. Math. Phys. 7 (1967), 200-217.

[3] Y. Censor, A. Lent, An iterative row-action method for interval convex programming, J. Optim. Theory Appl. 34 (1981), 321-358.

[4] G. Chen, M. Teboulle, Convergence analysis of a proximal-like minimization algorithm using Bregman functions, SIAM J. Optimization 3 (1993), 538-543.

[5] P. Hartman, G. Stampacchia, On some nonlinear elliptic differential functional equations, Acta Math. 115 (1966), 153-188.

[6] Ya. Alber, Metric and generalized projection operators in Banach spaces: properties and applications, in: A. Kartsatos (Ed.), Theory and Applications of Nonlinear Operators of Monotonic and Accretive Type, Marcel Dekker, pp. 15-50, New York, 1996.

[7] J. Li, J.-C. Yao, The existence of maximum and minimum solutions to general variational inequalities in Hilbert lattices, Fixed Point Theory Appl. 2011 (2011), 904320.

[8] T. Fujimoto, An extension of Tarski's fixed point theorem and its application to isotone complementarity problems, Math. Program. 28 (1984), 116-118.

[9] A. Chitra, P. Subrahmanyam, Remarks on nonlinear complementarity problem, J. Optim. Theory Appl. 53 (1987), 297-302.

[10] X. Qin, Y.J. Cho, S.M. Kang, Convergence theorems of common elements for equilibrium problems and fixed point problems in Banach spaces, J. Comput. Appl. Math. 225 (2009), 20-30.

[11] H. Nishimura, E.A. Ok, Solvability of variational inequalities on Hilbert lattices, Math. Oper. Res. 37 (2012), 559-674.

[12] L.C. Ceng, A subgradient-extragradient method for bilevel equilibrium problems with the constraints of variational inclusion systems and fixed point problems, Commun. Optim. Theory 2021 (2021), 4.

[13] S. Reich, S. Sabach, A strong convergence theorem for a proximal-type algorithm in reflexive Banach spaces, J. Nonlinear Convex Anal. 10 (2009), 471-485.

[14] C.-T. Pang, E. Naraghirad, C.-F. Wen, Bregman $f$-projection operator with applications to variational inequalities in Banach spaces, Abstr. Appl. Anal. 2014 (2014), 594285.

[15] Q.L. Dong, S.H. Ke, S. He, X. Qin, A new relaxed projection and its applications, J. Nonlinear Funct. Anal. 2021 (2021), 19.

[16] J. Li, E. A. Ok, Optimal solutions to variational inequalities on Banach lattices, J. Math. Anal. Appl. 388 (2012), 1157-1165.

[17] J. Li, The generalized projection operator on reflexive Banach spaces and its applications, J. Math. Anal. Appl. 306 (2005), 55-71. 
[18] Ya. Alber, S. Reich, An iterative method for solving a class of nonlinear operator equations in Banach spaces, Panamer. Math. J. 4 (1994), 39-54.

[19] S. Reich, A limit theorem for projections, Linear Multilinear Algebra 13 (1983), 281-290.

[20] G. Isac, Complementarity Problem, in: Lecture Notes in Math., vol. 1528, Springer-Verlag, 1992.

[21] G. Isac, On the order monotonicity of the metric projection operator, In: S.P. Singh (Ed.), Approximation Theory, Wavelets and Applications, Kluwer, Dordrecht, 1995.

[22] E. Naraghirad, On Bregman best proximity points in Banach spaces, Numer. Funct. Anal. Optim. 38 (2017), 409-426.

[23] E. Naraghirad, Bregman best proximity points for Bregman asymptotic cyclic contraction mappings in Banach spaces, J. Nonlinear Var. Anal. 3 (2019), 27-44.

[24] M.-M. Wong, Q. H. Ansari, J.-C Yao, Existence of solutions of generalized variational inequalities in reflexive Banach spaces, Appl. Math. Lett. 22 (2009), 197-201.

[25] D. Butnariu and A. N. Iusem, Totally Convex Functions for Fixed Points Computation and Infinite Dimensional Optimization, Kluwer Academic Publishers, Dordrecht 2000.

[26] F. Kohsaka, W. Takahashi, Proximal point algorithms with Bregman functions in Banach spaces, J. Nonlinear Convex Anal. 6 (2005), 505-523.

[27] C. Zălinescu, Convex Analysis in General Vector Spaces, World Scientific Publishing Co. Inc., River Edge NJ, 2002.

[28] E. Naraghirad, C.J. Yao, Bregman weak relatively nonexpanseve mappings in Banach spaces, Fixed point Theory Appl. 2013 (2013), 141.

[29] L. Eslamizadeh, E. Naraghirad, Bregman common skew-attractive point theorems for semigroups of nonlinear mappings in Banach spaces, Appl. Set-Valued Anal. Optim. 2 (2020), 235-253.

[30] R. T. Rockafellar, On the maximal monotonicity of subdifferential mappings, Pacific J. Math. 33 (1970), 209-216.

[31] H. Gazmeh, E. Naraghirad, The split common null point problem for Bregman generalized resolvents in two Banach spaces, Optimization, 70 (2021), 1725-1758.

[32] E. Naraghirad, W. Takahashi, J.-C. Yao, Generalized retraction and fixed point theorems using Bregman functions in Banach spaces, J. Nonlinear and Convex Anal. 13 (2012), 141-156.

[33] M. Tamadoni Jahromi, E. Naraghirad, On the existence of solutions of variational inequalities using Bregman distances in Banach spaces, J. Nonlinear Convex Anal. to appear

[34] P. Meyer-Nieberg, Banach Lattices, Universitext, Springer, Berlin, Heidelberg, 1991. 